\title{
Uma análise do desempenho dos estados nordestinos na política de ciência, tecnologia e inovação entre 2000 e 2015
}

Bruno Setton Gonçalves

Doutorando pelo Programa de Pós-Graduação em Ciência da Propriedade Intelectual da Universidade Federal de Sergipe e Professor Adjunto III do Curso de Administração Pública da Universidade Federal de Alagoas.

bruno.setton@arapiraca.ufal.br

https://orcid.org/0000-0002-3866-1870

José Ricardo de Santana

Doutorado em Economia de Empresas pela Fundação Getúlio Vargas - SP e Professor Associado do Departamento de Economia e do Programa de Pós-Graduação em Ciência da Propriedade Intelectual da Universidade Federal de Sergipe.

Santana_josericardo@yahoo.com.br

\section{Editor Científico: José Edson Lara}

Organização Comitê Científico

Double Blind Review pelo SEER/OJS

Recebido em 05.10.2019

Aprovado em 06.12.2019

\footnotetext{
Este trabalho foi licenciado com uma Licença Creative Commons - Atribuição - Não Comercial 3.0 Brasil
} 


\title{
Uma análise do desempenho dos estados nordestinos na política de ciência, tecnologia e inovação entre 2000 e 2015.
}

\section{Resumo}

Objetivo do estudo: estudo é propor um indicador que permita analisar o desempenho dos estados do Nordeste quanto a execução dos investimentos públicos em Ciência, Tecnologia \& Inovação (CT\&I) durante o período de 2000 a 2015;

Metodologia/abordagem: foi adotado um modelo lógico dedutivo, que inclui variáveis sugeridas pela literatura e estabelece uma ordem de causalidade entre elas, onde o desempenho está representado pelos aspectos relativos aos esforços mais os aspectos relativos aos resultados; Originalidade/relevância: a construção de indicadores possibilita estimar a efetividade das políticas públicas referentes à produção de ciência, tecnologia e inovação. Os indicadores apontam, indicam, aproximam, traduzem em termos operacionais as dimensões sociais de interesse definidas a partir de escolhas teóricas ou políticas realizadas anteriormente.

Principais resultados: os resultados mostram de forma inter-regional a disposição dos investimentos públicos em CT\&I, possibilitando o posicionar os estados nordestinos em um ranking formado por três blocos distintos quanto ao tamanho de sua base cientifica e tecnológica.

Contribuições teóricas/metodológicas: As contribuições desse estudo estão relacionadas as discussões do estado da arte em que destacam o papel do Estado como um agente ativo e importante na promoção da CT\&I, uma vez que essas políticas desempenham um papel fundamental na garantia do desenvolvimento econômico e social de um país.

Palavras-chave: Desenvolvimento; Indicadores; Inovação; Investimentos.

\section{An analysis of the performance of northeastern states in science, technology and innovation policy between 2000 and 2015.}

\begin{abstract}
Objective of study: This study proposes an indicator to analyze the performance of the Northeast states regarding the execution of public investments in Science, Technology \& Innovation (CT\&I) during the period from 2000 to 2015;

Methodology / approach: a deductive logical model was adopted, which includes variables suggested in the literature and establishes a causal order between them, where performance is represented by aspects related to efforts plus aspects related to results;

Originality / relevance: the construction of indicators makes it possible to estimate the effectiveness of public policies related to the production of science, technology and innovation. The indicators point, indicate, approximate, translate into operational terms the social dimensions of interest defined from theoretical or political choices previously made.

Main results: the results show, inter-regionally, the disposition of public investments in ST\&I, making it possible to position the northeastern states in a ranking formed by three distinct blocks regarding the size of their scientific and technological base.

Theoretical / Methodological Contributions: The contributions of this study relate to state of the art discussions highlighting the role of the state as an active and important agent in
\end{abstract}


promoting ST\&I, as these policies play a key role in ensuring economic and social development. of a country.

Keywords: Development; Indicators; Innovation; Investments.

Un análisis del desempeño de los estados del noreste en las políticas de ciencia, tecnología e innovación entre 2000 y 2015

\section{Resumen}

Objetivo del estudio: Este estudio propone un indicador para analizar el desempeño de los estados del Nordeste con respecto a la ejecución de inversiones públicas en Ciencia, Tecnología e Innovación (CT\&I) durante el período de 2000 a 2015;

Metodología / enfoque: se adoptó un modelo lógico deductivo, que incluye variables sugeridas en la literatura y establece un orden causal entre ellas, donde el desempeño está representado por aspectos relacionados con los esfuerzos más aspectos relacionados con los resultados;

Originalidad / relevancia: la construcción de indicadores permite estimar la efectividad de las políticas públicas relacionadas con la producción de ciencia, tecnología e innovación. Los indicadores señalan, indican, aproximan, traducen en términos operativos las dimensiones sociales de interés definidas a partir de elecciones teóricas o políticas tomadas previamente.

Resultados principales: los resultados muestran, interregionalmente, la disposición de las inversiones públicas en ST\&I, lo que permite posicionar a los estados del noreste en un ranking formado por tres bloques distintos con respecto al tamaño de su base científica y tecnológica.

Contribuciones teóricas / metodológicas: las contribuciones de este estudio se relacionan con las discusiones de vanguardia que destacan el papel del estado como agente activo e importante en la promoción de ST\&I, ya que estas políticas desempeñan un papel clave para garantizar el desarrollo económico y social. de un país.

Palabras clave: desarrollo; Indicadores; Innovación; Inversiones.

\section{Introdução}

A maioria dos argumentos a favor da intervenção do Estado para o crescimento e o desenvolvimento negligenciam a inovação, tomando-a como algo natural ao processo de crescimento. No entanto há uma discussão menos ortodoxa que estabelece uma ligação direta entre o Estado e a inovação, onde o empreendedorismo não se resume apenas a start-ups, capital de risco e da vontade individual do empreendedor Mas sobre tudo envolve a disposição e a capacidade dos agentes econômicos de assumir o risco e a incerteza da inovação (Mazzucato, 2015).

Segundo Mazzucato, (2015) a maioria das inovações radicais, que alimentaram o capitalismo aponta para o Estado na origem dos investimentos, a exemplo das ferrovias, internet, nanotecnologia e indústria farmacêutica. O grande exemplo atual é o advento do iphone uma das grandes inovações do momento só foi possível porque as tecnologias como: Internet, GPS, Touch-screen e o comando de voz SIRI foram financiados pelo Estado Norte 
Americano através de suas agências como a Defense Advanced Research Projects Agency (DARPA), a Small Business Investment Company (SIBC) e Small Business Innovation Research (SBIR), ou seja, foi a "mão invisível” do Estado que fez essas inovações acontecerem.

O Estado moderno busca promover e articular as atividades cientifica e tecnológicas e o processo de inovação nacional por meio do planejamento, fomento e incentivo, dessas ações. Diante do exposto o presente artigo parte da hipótese de que o nível e a direção dos investimentos em inovação é o que impulsiona a capacidade de crescimento da economia.

$\mathrm{Na}$ tentativa de avaliar o desempenho desses investimentos públicos é necessária a construção de indicadores que possibilitem estimar a efetividade das políticas públicas referentes à produção de ciência, tecnologia e inovação (CT\&I) Os indicadores apontam, indicam, aproximam, traduzem em termos operacionais as dimensões sociais de interesse definidas a partir de escolhas teóricas ou políticas realizadas anteriormente. Eles se prestam a subsidiar as atividades de planejamento público e a formulação de políticas sociais nas diferentes esferas de governo, possibilitam o monitoramento das condições de vida e bem-estar da população por parte do poder público e sociedade civil e permitem o aprofundamento da investigação acadêmica sobre a mudança social (Jannuzzi, 2005).

Nesse sentido, o objetivo do presente estudo é propor um indicador que permita analisar o desempenho dos estados do Nordeste quanto a execução dos investimentos públicos em Ciência, Tecnologia \& Inovação (CT\&I) durante o período de 2000 a 2015. Para tal foi adotado um modelo lógico dedutivo, que inclui variáveis sugeridas pela literatura e estabelece uma ordem de causalidade entre elas, onde o desempenho está representado pelos aspectos relativos aos esforços mais os aspectos relativos aos resultados.

Para alcançar os objetivos propostos, o artigo encontra-se estruturado em cinco seções: além dessa breve introdução, a segunda seção aborda o processo de formação do sistema regional de inovação, a terceira e quarta seções apresentam, respectivamente a metodologia e os resultados obtidos e por fim, a quinta e última seção contempla as considerações finais à guisa de conclusão.

\section{Sistema Regional de Inovação}

As políticas de CT\&I desempenham um papel fundamental na garantia do desenvolvimento econômico e social de um país. O Estado busca promover e articular as 
atividades de CT\&I por meio do seu financiamento e da construção de um marco legal capaz de responder as demandas existentes.

As políticas públicas em CT\&I nos países desenvolvidos são focadas no fortalecimento do que a abordagem neoschumpeteriana denomina de Sistema Nacional de Inovação (SNI). Estes sistemas são definidos por Freman, (1995) como uma rede de relacionamento entre instituições do setor público e privado para o desenvolvimento de ações que fomentam a geração de novas tecnologias e a inovação. A literatura mostra como sistemas de inovação dos países desenvolvidos estimulam as empresas com capacidade de inovação no desenvolvimento de novos produtos (Bezerra, Borini, \& Maclennan, 2015; Ghosh \& Soete, 2006).

Entretanto a compreensão acerca do desenvolvimento tecnológico e da inovação passa pela discussão da economia do conhecimento a partir da distinção que ela faz do conhecimento tácito e o conhecimento codificado, onde o primeiro se caracteriza por ser pessoal e específico ao contexto, difícil de ser formulado e replicado, estando vinculado aos agentes ou instituições e somente por intermédio desses é que pode ser acessado e utilizado. Já o conhecimento codificado é despersonalizado, refere-se ao conhecimento transmissível na linguagem formal e sistemática sendo transformado em informação, sua utilização possui um caráter mais amplo e não está inteiramente associada a determinado agente ou determinada instituição. A codificação do conhecimento permite, expandir as modalidades de acesso a esse conhecimento, estabelecendo a condição necessária para sua mercantilização, conforme prevê a aplicação dos direitos de propriedade intelectual e industrial (Herscovici, 2007).

Estas características do conhecimento, em especial o de natureza tácita, justificam sua natureza localizada e eventualmente uso do instrumental analítico de Sistema Regional de Inovação (SRI) ou de Arranjos Produtivos Locais (APLs). Estes níveis de análise motivam um conjunto de políticas regionais e/ou locais que fomentem a inovação e a competitividade industrial com possibilidade de impacto econômico e social, principalmente para os países em desenvolvimento. Estes países apresentam grandes disparidades regionais e locais em termos de estruturas produtivas e sociais. As políticas devem ser capazes de promover processos de aprendizado e inovação induzindo a um processo de desenvolvimento local dinâmico e sustentável (Szapiro, Mattos, \& Cassiolato, 2017)

O Brasil com suas dimensões continentais, e uma grande assimetria regional, apresenta características e necessidades muito peculiares no desafio de distribuir os recursos para a política de CT\&I. As desigualdades regionais prejudicam a eficácia de políticas públicas uniformes, uma vez que nem todas as regiões são capazes de aproveitar as condições favoráveis 
das políticas nacionais, diante disso o SRI torna-se importante e necessário nesse cenário uma vez que este é derivado do SNI e operam segundo as características especificas de cada região do país (Araújo \& Oliveira, 2015).

Entretanto é preciso ter a percepção que os resultados dos investimentos em CT\&I ocorrem no médio e longo prazo, o que exige dos atores públicos e privados o entendimento que políticas econômicas de austeridade não prejudiquem o crescimento de longo prazo. Investimentos públicos em pesquisa, desenvolvimento e inovação (PD\&I) são indutores do crescimento da produtividade, gerando empregos bem remunerados e com mais efeitos multiplicadores do que outras despesas governamentais, por isso a importância de se garantir a estabilidade desses investimentos no longo prazo. É indispensável pôr a inovação no cerne da política de crescimento econômico, articulando com mais consistência a política fiscal e as políticas de inovação tecnológica (Mazzucato \& Penna, 2016).

A percepção dessas conexões é essencial para a eficiência de políticas de apoio à inovação tecnológica, a maior aplicação combinada ao menor nível possível de variação dos recursos disponíveis é fundamental para o êxito das estratégias de catching-up de países e regiões (Lopes, 2014).

Os países desenvolvidos têm fomentado essas interações com investimentos para o surgimento de médias e pequenas empresas de base tecnológica, estimulando a interação e facilitando a parceria entre universidade-empresas, No caso do Brasil, as políticas de CT\&I tem adotado um caráter menos linear de apoio à inovação, porém ainda muito centralizado nas ações do Estado. (Salerno \& Kubota, 2008)

O Brasil deu passos firmes na área de inovação a partir de 1999, seguindo muitas práticas de incentivos à Pesquisa e Desenvolvimento (P\&D) utilizadas nos países membros da Organization Economics for Co-operation and Development (OCDE), podendo-se destacar a criação da Lei de Inovação e da Lei do Bem. No entanto, registra-se que o Brasil possui um ritmo diferente e, obviamente, recursos inferiores aos aplicados por países da OCDE (Negri, Negri, \& Lemos, 2008) e com desempenho ainda modesto em algumas variáveis, como é o caso e patentes e de gastos em P\&D sobre o PIB (Negri, 2017.a; Negri, 2017.b).

Mesmo assim, o país apresentou crescimento de capital humano especializado (graduados, mestres e doutores) com o aumento de universidades, cursos de graduação e pósgraduação. Ainda no final dos anos 90 um novo modelo de financiamento e gestão de CT\&I começou a ser construído no Brasil. O então Ministério da Ciência e Tecnologia tomou o 
protagonismo de um extenso debate acerca dos rumos que o país iria adotar na política de CT\&I. Em 2001 surgiu o documento "Ciência, Tecnologia e Inovação: Desafios para a Sociedade Brasileira - Livro Verde". O documento trazia em sua essência a importância de se fortalecer a tríplice hélice, apontando para o estímulo do setor privado tanto na definição das prioridades como no financiamento da CT\&I nacional e reforçando a importância das Fundações de Amparo à Pesquisa (FAP's) na gestão de fomento das pesquisas científicas e tecnológicas no país (Fonseca, 2012).

As FAP's estão ligadas aos seus respectivos governos estaduais, sendo um dos principais agentes da execução das políticas públicas de CT\&I no âmbito estadual. Elas se enquadram em uma categoria especifica de Fundação, responsável por investir no desenvolvimento de pesquisas (artigos científicos), recursos humanos (bolsas) tecnologia (patentes) e inovação (transferência de tecnologia e crescimento econômico da região), exercendo um papel fundamental e necessário de apoio à pesquisa tecnológica e cientifica dos estados, através das parcerias como o Conselho Nacional de Desenvolvimento Cientifico e Tecnológico (CNPq), a Financiadora de Estudos e Projetos (FINEP), a Coordenação de Aperfeiçoamento de Pessoal de Nível Superior (CAPES) ${ }^{1}$, Ministério da Ciência, Tecnologia, Inovação e Comunicação (MCTIC) e demais instituições regionais. Porém, está implantação é um desafio num país com as dimensões do Brasil, para manter a uniformidade das políticas públicas (Araújo \& Oliveira, 2015; Silva, 2009).

No mesmo período ainda são criados no âmbito federal os Fundos Setoriais de apoio ao desenvolvimento científico e tecnológico com a missão de financiar projetos de pesquisa, desenvolvimento e inovação ampliando assim o sistema nacional de CT\&I Os recursos dos fundos advém por meio das contribuições que incidem diretamente sobre o faturamento de empresas e sobre o resultado da exploração de recursos naturais que pertencem à União, tais como "royalties", Contribuições de Intervenção no Domínio Econômico (CIDE), compensações financeiras, autorizações, licenças e empréstimos, uma vez recolhidas essas contribuições são transferidos para o Fundo Nacional de Desenvolvimento Científico e Tecnológico (FNDCT) responsável por gerir os fundos setoriais (Fonseca, 2012).

\footnotetext{
${ }^{1}$ O Programa de Apoio e Parceria da CAPES com as Fundações Estaduais de Amparo à Pesquisa - CAPES/FAPs, regulamentado pela Portaria Capes n $\mathrm{n}^{\circ}$ 106, de 13 de agosto de 2014 - tem o objetivo de conceder bolsas de estudo e financiar projetos de pesquisa, buscando atender as necessidades de cada Unidade da Federação parceira. Sendo atualmente mantidas parcerias com os governos estaduais por meio de 25 acordos de cooperação firmados com as FAPs dos seguintes estados: AC, AL, AM, AP, BA, CE, ES, GO, MA, MG, MS, MT, PA, PB, PE, PI, PR, RJ, RN, RO, RS, SC, SE, SP e TO
} 
Esta evolução resultou numa maior produção de artigos científicos e de patentes. Além do estimulo a distribuição entre as federações destes investimentos. Porém, proporcionalmente o capital humano ainda não é suficiente para atender todas as regiões do país, o que representa um gargalo a ser superado uma vez que são os níveis dos investimentos em CT\&I que determinam a escolha do processo de desenvolvimento que as regiões irão adotar e, sobretudo, na maneira pela qual essa escolha não só impacta como também modificará a qualidade de vida da sociedade (Asheim \& Isaksen, 2002).

O governo federal é o responsável por distribuir os recursos e pacotes de incentivos para as suas federações de maneira proporcional (tamanho da região e capacidade econômica). No entanto, cada estado possui o seu sistema local de inovação para fomentar financeiramente a CT\&I na sua região. Para estimular isto a nível regional os governos tem que combinar os instrumentos das políticas públicas cuidadosamente para atender os problemas complexos do processo de inovação (Borrás \& Edquist, 2013).

A intensificação dos investimentos públicos em CT\&I poderá garantir, a infraestrutura cientifica e tecnológica necessária aos estados de menor dinamismo econômico. No entanto as ações dos estados não devem se limitar somente a garantir maiores volumes de investimento é preciso aprimorar continuamente sua intervenção de forma a garantir o nível de recursos alocados na política pública, no intuito de induzir o setor privado a um investimento mais significativo em inovação tecnológica nas diferentes regiões. Para tal é preciso que o papel da intervenção estatal na economia seja repensado para além das falhas de mercado e nesse sentido a política pública de CT\&I, desempenha o papel de indutora de novos padrões de produção, distribuição e consumo (Mazzucato, 2015).

\section{Procedimentos metodológicos}

Serão expostos aqui os métodos e técnicas utilizados para a construção do indicador que permita avaliar o nível de investimento público estadual em CT\&I e seus impactos no número de registro de patentes e do número de doutores do Nordeste no período de 2000 a 2015.

\subsection{Modelo}

A pesquisa fez uso de um recurso analítico com o propósito de representar a realidade a partir da definição de um conjunto de variáveis (ou aspectos da realidade que se pretende 
representar), aspectos estes que podem ser vistos/definidos/medidos de forma quantitativa por meio de indicadores.

A escolha das variáveis e seus padrões (hipóteses) de relacionamento (causação) podem seguir várias lógicas: relações causais verificadas empiricamente ou lógica dedutiva. (Ferreira, Cassiolato, \& Gonzalez, 2007). O presente artigo, portanto, adotou um modelo lógico dedutivo, que inclui variáveis sugeridas pela literatura e estabelece uma ordem de causalidade entre elas, onde o desempenho está representado pelos aspectos relativos aos esforços mais os aspectos relativos aos resultados. A equação simplificada é:

\section{Desempenho $=$ esforços + resultados}

Nesse sentido a forma funcional do indicador proposto é dada por:

$$
\mathrm{Id}=\frac{(\text { disp } / P I B)}{(\mathrm{n} \text { - de Doutores/patentes })}
$$

Sendo:

Id: é o índice de desempenho para cada estado; Disp: é o dispêndio público estadual em CT\&I; PIB: Produto Interno Bruto estadual; $\mathrm{N}^{\circ}$ de doutores: contabilizados no último censo da CAPES por estado; Patentes: depósito de patentes por estado.

De modo que a relação dispêndio/PIB representa o esforço e a relação doutores/patentes o resultado. $\mathrm{O}$ valor compreende entre 0 e 1 , onde 1 representa o maior desempenho e 0 o menor.

\subsection{Base de dados e descrição das variáveis}

Com a escolha assume-se que a realidade é um todo complexo e o modelo, ao incluir apenas algumas variáveis para representá-la (buscando descrever, explicar ou prever algo), é limitado, ao deixar de fora muitas variáveis potencialmente importantes para estabelecer os padrões de comportamento entre as variáveis. No entanto essa limitação foi minorada por meio da escolha das variáveis entendidas como as mais relevantes.

\section{Tabela 1}

Matriz Síntese do Modelo de Medição do Desempenho

\begin{tabular}{|c|l|c|}
\hline Tipo Variável & \multicolumn{1}{|c|}{ Descrição } & Fonte \\
\hline \multirow{2}{*}{ Variáveis de Insumo } & PIB estadual & IPEA \\
\cline { 2 - 3 } & $\begin{array}{l}\text { Volume dos gastos públicos } \\
\text { Estaduais em CT\&I }\end{array}$ \\
\hline Variáveis de Resultado & Registro de patentes & MCTI e INPI \\
\cline { 2 - 3 } & Número de doutores & CNPQ \\
\hline
\end{tabular}

Fonte: Elaboração dos autores. 
Segundo Rua (2004), Jannuzzi (2005) e Santagada (2007) os indicadores de insumo (antes) são aqueles que possuem relação direta com os recursos a serem alocados, no modelo proposto eles são apresentados pelo volume da despesa orçamentária alocada na função de CT\&I dos estados e regiões. Já os indicadores de resultado (depois) expressam, direta ou indiretamente, os benefícios no público-alvo, que no presente artigo estão representados pelo registro de depósito de patentes, que é composto pelo somatório das patentes de invenção, dos modelos de utilidade e dos certificados de adição. Além do número de doutores por habitantes segundo região e unidade da federação que na proposta apresentada as unidades federativas são as da região Nordeste.

O que se espera é que ocorra uma relação causal positiva entre os indicadores de insumo e os de resultado, onde, uma maior alocação de recursos orçamentário em CT\&I proporcione um maior número de pesquisadores/doutores e logo um maior índice de registro de patentes, o que em tese sinalizaria uma economia mais inovadora.

\section{Apresentação e discussão dos resultados}

$\mathrm{Na}$ perspectiva que os investimentos em CT\&I contribuem para o aumento da competitividade de países e regiões e logo para o crescimento econômico, conforme apontado pela literatura abordada. E de que essas condições são propiciadas dentro do Sistema Nacional de Inovação (SNI), através do modelo tríplice hélice, onde há uma sinergia entre os atores envolvidos (Empresas, Universidades e Governo). Onde os governos tem fundamentalmente o papel do fomentar e induzir, o comportamento, as estratégias e as decisões empresariais relativas à inovação.

Nesse sentido o intuito aqui é apresentar um indicador que permita identificar o desempenho dos estados nordestinos na execução da política pública de CT\&I. Para isso o presente artigo partirá da visão de Rua (2004), onde os indicadores são medidas que expressam ou quantificam um insumo, um resultado, uma característica ou o desempenho de um processo, serviço, produto ou organização. E Magalhães (2004), para o qual indicadores são abstrações ou parâmetros representativos, concisos, fáceis de interpretar e de serem obtidos, usados para ilustrar as características principais de determinado objeto de análise. 


\section{1 $\mathrm{O}$ indicador de Insumo}

A primeira relação é expressa através de um indicador de insumo apresentado pela razão (dispêndio/PIB) representando os aspectos relativos aos esforços desprendidos pelos estados para a execução da política pública de CT\&I. Esse indicador é apresentado na figura 1, a qual traz o ranking por média dos dispêndios em CT\&I dos Estados do Nordeste em relação aos seus respectivos PIB's.

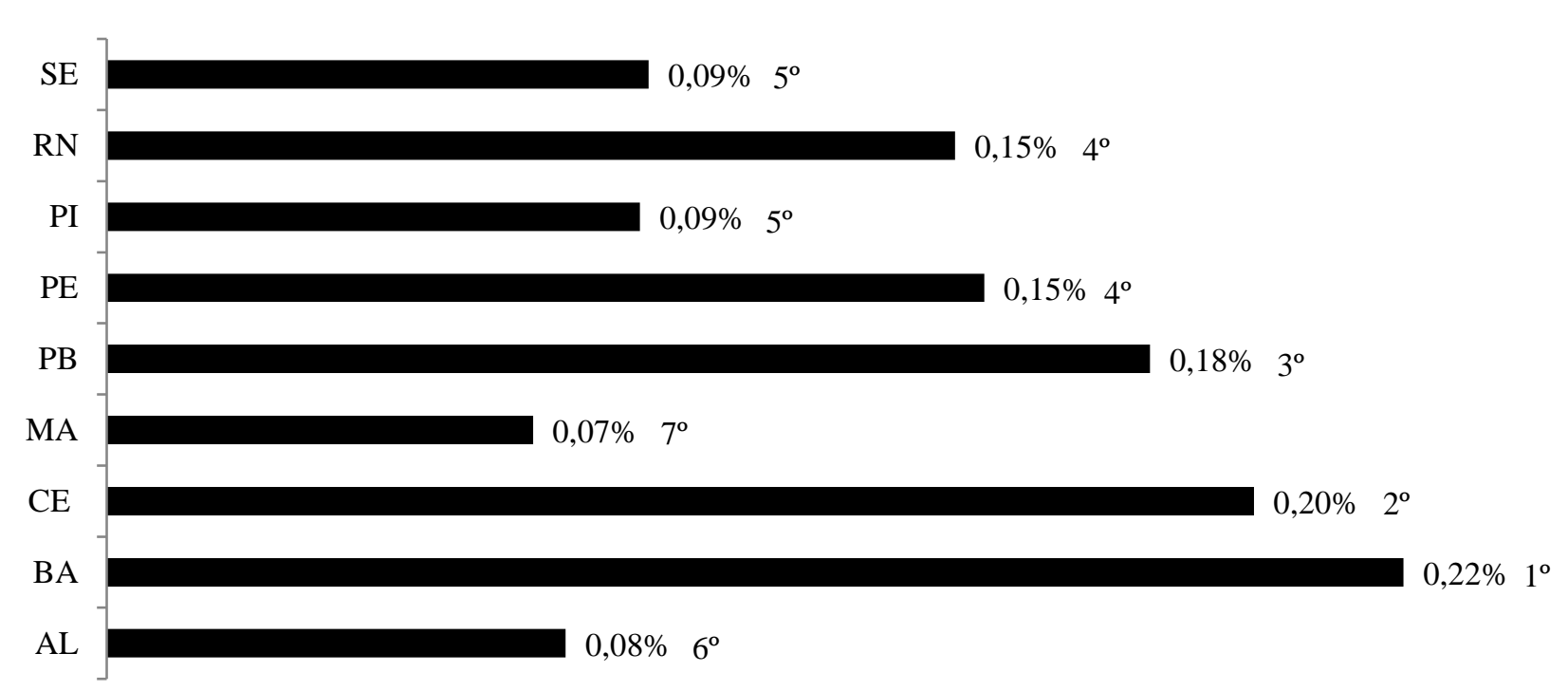

Figura 1: Ranking por Média dos Dispêndios em CT\&I dos Estados do NE em relação ao PIB (2000-20130

Fonte: Brasil (2018), Ministério da Ciência, Tecnologia, Inovações e Comunicações e Ipea (2018).

É possível constar na figura 1 que os estados nordestinos ainda destinam muito pouco dos seus orçamentos públicos para a função de CT\&I, em média a região tem disponibilizado apenas $0,14 \%$ do PIB regional para a execução da política. Esse percentual coloca e região Nordeste na terceira posição entre as regiões com um dispêndio médio de R\$ 700 milhões durante a série apresentada, o Sudeste que desponta na primeira colocação em nível de gasto apresenta uma média de R \$ 5.077 bilhões para o mesmo período, conforme figura 2. 


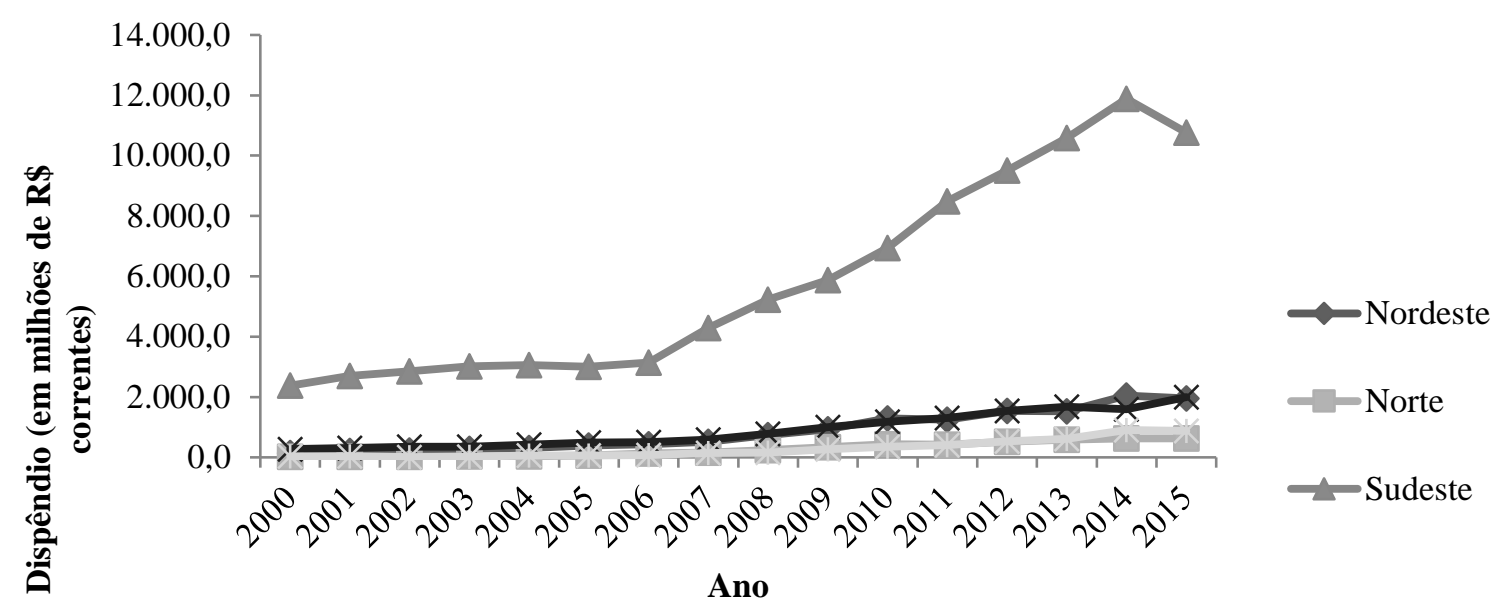

Figura 2: Dispêndios Públicos em Ciência e Tecnologia, por região (2000-2015) (em R\$ milhões)

Fonte: Brasil (2018), Ministério da Ciência, Tecnologia, Inovações e Comunicações.

Os dados de dispêndio em C\&T mostram uma tendência de crescimento, entre 2000 e 2015, sendo mais acentuado na região Sudeste, como apresentado na Figura 2. A figura ainda demostra o alto grau de assimetria regional ao explicitar o hiato que existente entre o Sudeste e as demais regiões. Essa desigualdade também está presente entre as demais regiões basta comparar as regiões Sul e Nordeste, a primeira com somente três estados consegue apresentar um volume de dispêndio ligeiramente superior ao do Nordeste com nove estados, o quadro 1 evidencia essa desigualdade quando compara os valores correntes de 2015 dos três estados do Sul com os três estados do Nordeste que mais dispendem recursos públicos para C\&T.

Tabela 2

Dispêndios dos governos estaduais em (C\&T) em 2015 (em milhões de R \$ correntes)

\begin{tabular}{|l|c|l|c|}
\hline \multicolumn{1}{|c|}{ Estados do Sul } & Dispêndio & \multicolumn{1}{c|}{ Estados do Nordeste } & Dispêndio \\
\hline Paraná & 948,3 & Bahia & 780,2 \\
\hline Rio Grande do Sul & 413,1 & Ceará & 271,8 \\
\hline Santa Catarina & 636,1 & Paraíba & 215,5 \\
\hline
\end{tabular}

Fonte: Brasil (2018), Ministério da Ciência, Tecnologia, Inovações e Comunicações.

A Bahia que é o estado com o maior volume de gastos em C\&T do Nordeste, representa apenas $82 \%$ do volume de gastos do Paraná que é o estado com maior nível de gastos na função da região Sul. O Ceará apresenta $65 \%$ dos gastos do Rio Grande Sul e a Paraíba apenas 34\% dos dispêndios de Santa Catarina. 
Tabela 3

Comparação dos dispêndios em CT\&I no Brasil entre os setores (em valores de 2015) com o produto interno bruto (PIB)

\begin{tabular}{c|c|c|c}
\hline \multirow{2}{*}{$\%$ PIB } & \multirow{2}{*}{$\begin{array}{c}\text { \%PIB gasto pelas } \\
\text { empresas }\end{array}$} & \multicolumn{2}{|c}{ \% do PIB gasto público } \\
\cline { 3 - 4 } & 0,64 & \% do PIB Governo Federal & \% do PIB Governos Estaduais \\
\hline 1,28 & & 0,45 & 0,19 \\
\hline
\end{tabular}

Fonte: Brasil, (2018), Coordenação de Indicadores e Informação (COIND) - CGGI/DGE/SEXEC - Ministério da Ciência, Tecnologia, Inovações e Comunicações (MCTIC).

Os resultados encontrados para os estados nordestinos no período analisado, demostram estar coerentes com a execução da política nacional de CT\&I, conforme demostra a tabela 1 o país destinou em valores de 2015 1,28\% do seu PIB para o fomento da política, onde os estados participaram em média com $0,19 \%$, um percentual um pouco acima do encontrado para os estados do Nordeste, $0,14 \%$.

\subsection{O indicador de Resultado}

Diante do esforço apresentado pelos estados segue agora o indicador que expressa os resultados decorrentes da alocação dos recursos. Esse indicador apresentado na figura 3 demostra a relação entre o número de doutores pesquisadores e o registro de deposito de patentes em cada estado do Nordeste para o ano de 2015.

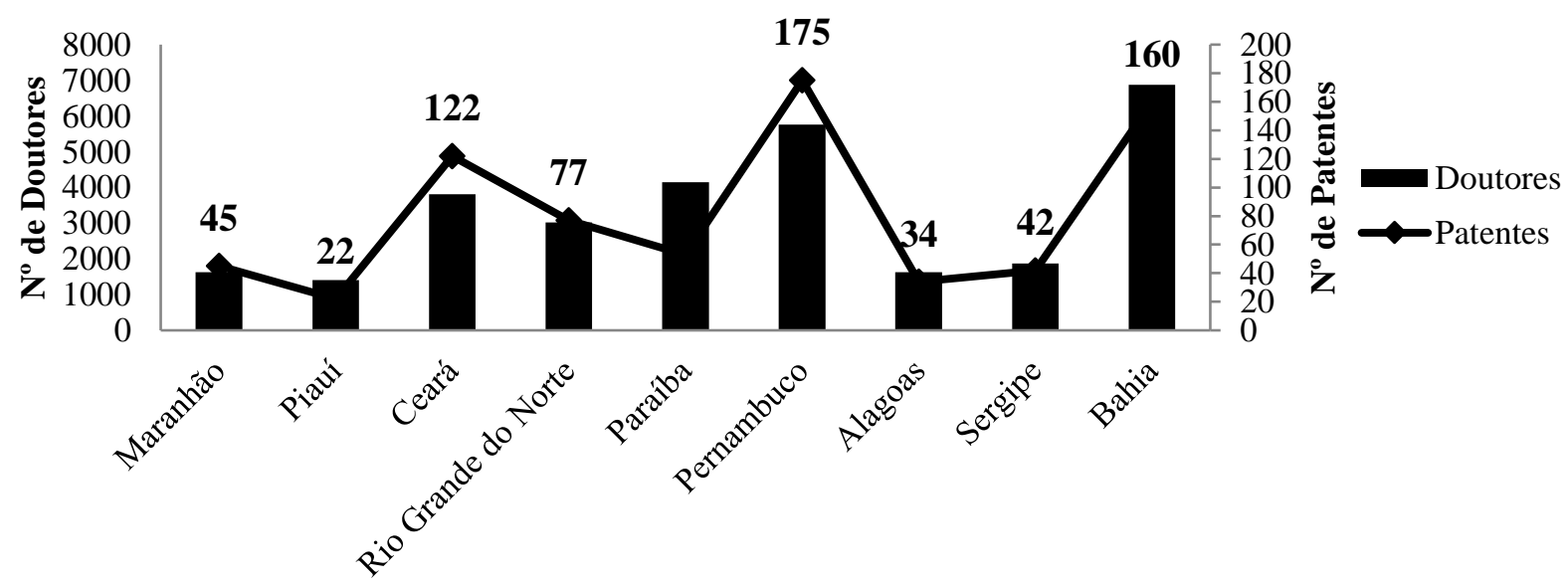

Estados

Figura 3: Relação entre o número de doutores e o depósito de patentes 2015 Fonte: (Inpi, 2018); (CNPq, 2018).

A relação doutores e depósito de patentes expresso na figura 2 destacam os estados da Bahia, Ceará e Pernambuco bem à frente dos demais estados da região. Dentre os três 
destacados vale ressaltar o desempenho do estado de Pernambuco que com um número menor de pesquisadores doutores (5.761) em relação à Bahia (6.870) consegue registrar um maior número de patentes junto ao instituto nacional de Propriedade Industrial (INPI) em 2015.

Uma expressiva parte desses números apresentados pelos estados do Nordeste é induzida por programas do $\mathrm{CNPq}$ como o Programa Institucional de Bolsas de Iniciação em Desenvolvimento Tecnológico e Inovação (PIBITI) que tem por objetivo estimular os jovens do ensino superior nas atividades, metodologias, conhecimentos e práticas próprias ao desenvolvimento tecnológico e processos de inovação. O programa em 2015 destinou 3142 bolsas, o Nordeste foi contemplado com 580 bolsas $(18,46 \%)$ só ficando atrás das regiões Sudeste $(41,02 \%)$ e $\operatorname{Sul}(28,17 \%)$.

Cabe ressaltar que as grades áreas beneficiadas pelo programa no Nordeste foram às engenharias $(34,14 \%)$, ciências agrarias $(17,95 \%)$ e ciências exatas e da terra com $(17,59 \%)$ das bolsas (CNPq, 2018).

\subsection{O indicador de Desempenho}

O desempenho é representado pelos aspectos relativos aos esforços mais resultados, onde a relação dispêndio/PIB representa o esforço e a relação doutores/patentes o resultado. Onde valores mais próximos de 1 indica um maior desempenho do estado na execução da política pública de CT\&I.

Fazendo um exercício de simplificação, considerando que a base de CT\&I estivesse resumida as variáveis, registros de patentes e número de doutores pesquisadores. O Nordeste poderia ser dividido em três blocos distintos quanto ao tamanho de sua base cientifica e tecnológica, onde o primeiro bloco seria composto por Bahia, Ceara e Pernambuco, o segundo formado por Rio Grande do Norte e Paraíba e o terceiro pelo Maranhão, Sergipe, Alagoas e o Piauí.

Contudo ao se aplicar o indicador de desempenho para os estados do Nordeste é possível redimensionar essa configuração do ranking posicionando cada estado quanto ao seu real desempenho na execução da política local de CT\&I, conforme demostra o quadro 2.

\section{Tabela 4}

Ranking por Desempenho dos Estados Nordestinos 


\begin{tabular}{|c|c|c|c|c|}
\hline \multicolumn{5}{|c|}{ Indicador } \\
\hline UF & CT\&I / PIB & Doutores/Patentes & Id & Ranking \\
\hline CE & 0,20 & 29 & 0,69 & $1^{\mathbf{o}}$ \\
\hline BA & 0,22 & 35 & 0,64 & $2^{\mathbf{o}}$ \\
\hline RN & 0,15 & 46 & 0,33 & $3^{\mathbf{o}}$ \\
\hline PE & 0,15 & 46 & 0,33 & $4^{\mathbf{o}}$ \\
\hline PB & 0,18 & 73 & 0,25 & $5^{\mathbf{o}}$ \\
\hline MA & 0,007 & 30 & 0,02 & $6^{\mathbf{o}}$ \\
\hline SE & 0,009 & 42 & 0,02 & $7^{\mathbf{0}}$ \\
\hline AL & 0,008 & 38 & 0,02 & $8^{\mathbf{o}}$ \\
\hline PI & 0,009 & 62 & 0,01 & $9^{\mathbf{o}}$ \\
\hline
\end{tabular}

Fonte: Elaborado pelos autores

É possível perceber que apesar da Bahia possuir uma relação investimento/PIB maior do que o Ceará, este consegue ter uma melhor eficiência na produção de patentes, com uma relação de 29 doutores para cada patente depositada, enquanto a Bahia apresenta uma relação de 35 pesquisadores para cada patente registrada. O mesmo vale para a Paraíba em relação aos estados de Pernambuco e do Rio Grande do Norte. A Paraíba possui uma relação investimento/PIB maior que seus vizinhos, no entanto seu desempenho na relação doutores por patentes depositadas faz com que seu indicador de desempenho posicione o estado, na $5^{\text {a }}$ colocação no ranking.

\section{Tabela 5}

Classificação do Desempenho dos Estados Nordestinos por Grupo

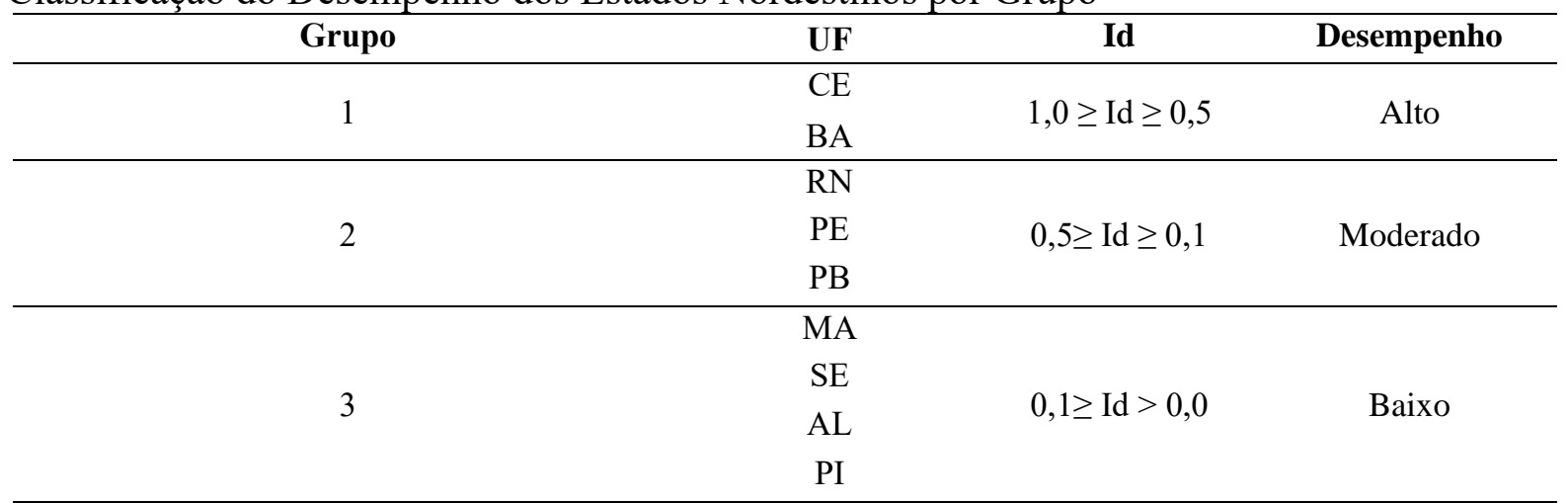

Fonte: Elaborado pelos autores

Essa nova perspectiva criada após a aplicação do indicador de desempenho traz uma nova reconfiguração do cenário conforme demostra a tabela 2 , onde o primeiro bloco fica constituído pelos estados que possui um indicador igual e/ou superior a 0,5 (Ceará e Bahia), o segundo bloco formado por estados com indicador igual e/ou superior a 0,1 (Rio Grande do Norte, 
Pernambuco e Paraíba) e o terceiro pelos estados com indicador menor que 0,1 (Maranhão, Sergipe, Alagoas, Piauí).

\section{Considerações finais}

Seguem nesta secção algumas considerações finais à guisa de conclusão, a primeira consideração a ser observada é que o nível de investimento do Nordeste é muito baixo, a média dos nove estados não ultrapassa $0,14 \%$ do PIB regional para a execução da política. Percentual abaixo da média dos estados brasileiros que participaram em média com $0,19 \%$.

Quanto aos resultados provenientes desse esforço depreendido pelos estados nordestinos tem-se a relação entre o número de doutores pesquisadores e o registro de deposito de patentes para cada estado do Nordeste no período de 2015. Para este indicador de resultados destacamse os estados da Bahia, Ceará e Pernambuco bem à frente dos demais estados da região. Dentre os três destacados vale ressaltar o desempenho do estado de Pernambuco que com um número menor de pesquisadores em relação à Bahia consegue registrar um maior número de patentes junto ao instituto nacional de Propriedade Industrial (INPI) em 2015.

É importante destacar que uma significativa parte desses números apresentados pelos estados do Nordeste é induzida por programas do CNPq como o Programa Institucional de Bolsas de Iniciação em Desenvolvimento Tecnológico e Inovação (PIBITI), o Nordeste foi contemplado com $18,46 \%$ das bolsas em 2015. Onde as grades áreas beneficiadas pelo programa na região foram às engenharias $(34,14 \%)$, ciências agrarias $(17,95 \%)$ e ciências exatas e da terra com $(17,59 \%)$ das bolsas.

No entanto o indicador de desempenho demostra que somente a relação esforço não é suficiente para garantir um bom desempenho na execução da política pública de CT\&I. O que indica haver outros componentes para além do financiamento que garantem o bom desempenho, essa constatação fica evidente ao observar o desempenho da Paraíba com relação a Pernambuco e ao Rio Grande do Norte e o da Bahia em relação ao do Ceará.

Nesses casos é possível perceber que tanto a Paraíba quanto a Bahia possuem um nível de investimento maior, contudo os mesmos apresentam um desempenho menor que os estados de Pernambuco e Rio Grande do Norte e do Ceará respectivamente.

Ao se aplicar o indicador de desempenho para os estados do Nordeste é possível posicionar cada estado quanto ao seu real desempenho na execução da política local de CT\&I. Onde o primeiro bloco fica constituído pelos estados que possui um alto desempenho (Ceará e 
Bahia), o segundo bloco formado por estados com um desempenho moderado (Rio Grande do Norte, Pernambuco e Paraíba) e o terceiro pelas unidades federativas com baixo desempenho (Maranhão, Sergipe, Alagoas, Piauí).

Por fim vale ressaltar as limitações desse trabalho, que apesar de ter identificado de forma inter-regional a disposição dos investimentos públicos em CT\&I, possibilitando o posicionar os estados nordestinos em um ranking, não nos permite inferir o quanto desse esforço dos estados (dispêndio em P\&D) tem impactado no crescimento e desenvolvimento econômico desses estados para tal será necessário ampliar a agenda de pesquisa para o desenvolvimento de um modelo que correlacione essas variáveis de forma a se obter essa relação de causalidade.

\section{Referências Bibliográficas}

Araújo, M. S., \& Oliveira, E. C. (13 e 14 de Agosto de 2015). As Características das FAPS e Atuação da FAPESC como Agente de Fomento no Sistema Regional de Inovação. XI Congresso Nacional de Excelência em Gestão. Brasil.

Asheim, B. T., \& Isaksen, A. (2002). Regional Innovation Systems: The Integration of Local Sticky and Global Ubiquitous Knowledge. Journal of Technology Transfer, 27, pp. 77-86.

Bezerra, M. A., Borini, F. M., \& Maclennan, M. L. (2015). Ferranty Reverse Transfers of Innovation and National Development: Evidence from Brazilian Subsidiaries. $J$. Technol Manag Innov, 10.

Borrás, S., \& Edquist, C. (2013). The choice of innovation policy instruments. Techological Forecasting and Social Change, 80(8), pp. 151-322.

Brasil. (2018). Ministério da Ciência, Tecnologia, Inovações e Comunicações (MCTIC). Acesso em 17 de Abril de 2018, disponível em MCTIC: http://www.mctic.gov.br

CNPq. (2018). Conselho Nacional de Desenvolvimento Científico e Tecnológico (CNPq). Acesso em 23 de Março de 2018, disponível em Sitio do CNPq: http://www.cnpq.br/indicadores1

Ferreira, H., Cassiolato, M., \& Gonzalez, R. (2007). Como Elaborar Modelo Lógico de Programas: um roteiro básico. Nota Técnica, IPEA.

Fonseca, M. L. (2012). Formulação de Políticas Públicas de Ciência, Tecnologia e Inovação. V Congresso CONSAD de Gestão Pública. Brasília.

Freman, C. (1995). The National System of Innovation in historical perspective. Cambridge Journal of Economics.

Ghosh, R., \& Soete, L. (2006). Information and intellectual property: the global challenges. Industrial and Corpoate Change, 15(6), pp. 919-935.

Herscovici, A. (2007). Capital intangível e direitos de propriedade intelectual: uma análise institucionalista. Revista de Economia Política, 27, pp. 394-412.

Inpi. (2018). Instituto Nacional de Propriedade Industrial (INPI). Acesso em 23 de Março de 2018, disponível em Sitio do INPI: http://www.inpi.gov.br/menuserviços/patente/guia-basico-de-patente 
Ipea. (2018). Instituto de Pesquisa Aplicada (IPEA). Acesso em 23 de Março de 2018, disponível em Sitio do IPEA: http://www.ipeadata.gov.br/Default.aspx

Jannuzzi, P. d. (2005). Indicadores para Diagnóstico, Monitoramento e Avaliação de Programas Sociais no Brasil. Revista do Serviço Público, 56, pp. 137-160.

Lopes, H. C. (2014). Os determinantes do desenvolvimento (catching up) na abordagem neoschumpeteriana: integrando a teoria microeconômica evolucionária com conceito de revoluções tecnológicas. Nexos Econômicos, 8.

Magalhães, M. (2004). Metodologia para Desenvolvimento de Sistemas de Indicadores: Uma Aplicação no Planejamento e Gestão da Política Nacional de Transportes. Dissertação. Brasília, Brasil: UNB.

Mazzucato, M. (2015). O Estado Empreendedor: desmascarando o mito do setor público vs. setor privado (1 ed.). (E. Serapicos, Trad.) São Paulo: Portifolio-Penguim.

Mazzucato, M., \& Penna, C. (2016). The Brazilian Innovation System: A Mission-Oriented Policy Proposal. Sumário Executivo. Avaliação de Progamas em CT\&I, apoio ao Programa Nacional de Ciência, Centro de Gestão e Estudos Estratégicos (CGEE), Brasília.

Negri, F. d. (2017.a). Políticas de Apoio à Inovação Tecnologica no Brasil: avanços recentes, limitações e propostas de ações. Brasília: IPEA.

Negri, F. d. (2017.b). Por uma Nova Geração de Políticas de Inovação no Brasil. Em L. M. TURCHI, \& J. M. MORAIS, Políticas de Apoio à Inovação Tecnológica no Brasil: avanços recentes, limitaçoes e propostas de ações (p. 485). Brasília: IPEA.

Negri, J. A., Negri, F. d., \& Lemos, M. B. (2008). O impacto do FNDCT sobre o desempenho e o esforço tecnológico das empresas e industriais brasileiras. (IPEA, Ed.) Textos para Discusão.

Rua, M. d. (2004). Desmistificando o problema: uma rápida introdução ao estudo dos indicadores. Mimeo.

Salerno, M., \& Kubota, L. C. (2008). Estado e Inovação. IPEA.

Santagada, S. (2007). Indicadores Socias: uma primeira abordagem social e histórica. Revista Pensamento Plural, 1, pp. 113-142.

Silva, F. Q. (2009). Um modelo de desenvolvimentolocal baseado em inovação e o papel dos parques tecnólogicos na sua implatação. Revista da Micro e Pequena Empresa, 3, pp. 25-37.

Szapiro, M., Mattos, M., \& Cassiolato, J. (2017). Sistemas de Inovação e Desenvolvimento. Em M. S. RAPINI, L. A. SILVA, \& E. d. ALBUQUERQUE, Economia da Ciência, Tecnologia e Inovação: Fundamentos Teóricos e a Economia Global (pp. 371-412). Curitiba: Prismas. 